\title{
El uso de fuentes documentales y orales en la investigación
arqueológica. El caso de El Siasgo, Depresión del río Salado
}

\author{
( Virginia Mariana Salerno* \\ Paula Escosteguy*** \\ Olivia Sokol ${ }^{* * *}$
}

Fecha de recepción: 28 de noviembre de 2018. Fecha de aceptación: 15 de junio de 2019

Palabras clave

arqueología pampeana memoria indígena patrimonio Ameghino

\section{Resumen}

Desde una perspectiva territorial, en este trabajo analizamos el potencial de integrar diversas fuentes escritas y orales en la contextualización de una investigación arqueológica. Para ello, presentamos el caso del arroyo El Siasgo de la Depresión del río Salado bonaerense. Identificamos y sistematizamos múltiples fuentes vinculadas con el pasado investigado: documentos sobre investigaciones arqueológicas previas, memorias de museos, correspondencia científica, material cartográfico y documentos del siglo XVIII y XIX. Además, incluimos testimonios orales relacionados con los vestigios arqueológicos. En conjunto, estas fuentes ofrecen información sobre las transformaciones de El Siasgo como un espacio construido estratégicamente a lo largo del tiempo. Su estudio integrado da cuenta de las diversas trayectorias poblacionales que se entrelazan en el Siasgo y que, en parte, son referidas en las representaciones que se construyen sobre los objetos arqueológicos.

The use of written and oral sources in archaeological research. The case of El Siasgo, Salado River Depression

\section{Abstract}

Keywords

Pampean archaeology indigenous memory heritage Ameghino

\footnotetext{
* Consejo Nacional de Investigaciones Científicas y Técnicas (CONICET) - Instituto de Arqueología, Facultad de Filosofía y Letras (FFyL), Universidad de Buenos Aires (UBA). Temperley, Ciudad Autónoma de Buenos Aires, Argentina. E-mail: vmasalerno@gmail.com

** CONICET -Instituto de Arqueología, FFyL, UBA. Tristán Suárez, Ciudad Autónoma de Buenos Aires, Argentina. E-mail: paueguy@hotmail.com

*** Instituto de Arqueología, FFyL, UBA. Ciudad Autónoma de Buenos Aires, Argentina. E-mail: olivia.l.sokol@ gmail.com
}

In this work we analyze the potential of integrating various written and oral sources in the contextualization of an archaeological research project from a territorial perspective. With this goal in mind, we present a case study of 
El Siasgo creek in the Salado River Depression of Buenos Aires. We identify and systematize multiple sources linked to the researched past: documents about previous archaeological research, museum memorials, scientific correspondence, cartographic material and documentary sources from the 18th and 10th centuries. In addition, we also include oral testimonies related to the archaeological remains. Combined, these sources offer data on the transformations of El Siasgo as a strategically built space over time, accounting for the trajectories of different populations that are interwoven together in El Siasgo and that are referred to, in part, in the representations of the archaeological objects.

\section{Introducción}

En este trabajo discutimos la integración de diversas fuentes escritas y orales relacionadas -directa o indirectamente- con los procesos poblacionales que estudiamos en la Depresión del río Salado bonaerense. Nuestra estrategia articula la búsqueda de nuevas evidencias arqueológicas de momentos prehispánicos con el estudio de los modos en que la arqueología es parte del presente en los ámbitos locales. Como caso de estudio presentamos las investigaciones arqueológicas en el sector de El Siasgo, donde el arroyo homónimo desemboca en el río Salado, en la intersección de los partidos de General Paz y San Miguel del Monte, provincia de Buenos Aires. Este es un paraje rural muy cercano al pueblo de Villanueva -a $5 \mathrm{~km}$ aproximadamente-, en el cual se contabilizaron 537 habitantes en el último censo (INDEC, 2012). En esta zona iniciamos investigaciones arqueológicas sistemáticas en el año 2013, en continuidad con los trabajos que se realizan en la microrregión desde 1986 (González, 2005; entre otros). Estos estudios evidenciaron numerosas ocupaciones asignadas al Holoceno tardío, lo que indica que el paisaje del río Salado y sus lagunas asociadas fueron ocupados reiteradamente por poblaciones de cazadores-recolectores-pescadores.

Para contextualizar nuestro problema de investigación identificamos y sistematizamos variados registros: documentos sobre investigaciones arqueológicas previas, memorias de museos, correspondencia científica, material cartográfico y documentos del siglo XVIII y XIX. Además, incluimos testimonios orales relacionados con los vestigios arqueológicos. En este espacio, reflexionamos sobre el modo en que estas diversas fuentes enriquecen nuestra perspectiva de los problemas arqueológicos que estamos abordando.

\section{Consideraciones metodológicas}

Utilizamos la noción de "fuente" en sentido amplio, que incluye testimonios orales así como materiales publicados e información inédita -ya sea que no fue publicada previamente o que no está destinada a publicarse- (Nacuzzi y Lucaioli, 2011). En cuanto a las fuentes escritas, su aporte y articulación con el estudio de los restos materiales ha sido ampliamente debatido en las investigaciones de arqueología histórica. ${ }^{1}$ De estos debates, rescatamos la valoración de los documentos escritos como una fuente de información que, al igual que los restos materiales, pueden analizarse para abordar problemas relativos al pasado que se busca investigar. Para este estudio, recuperamos los desafíos metodológicos planteados por Nacuzzi y Lucaioli (2011) referidos tanto a la fragmentación y disponibilidad de las fuentes, como a la posibilidad de interpretar las acciones y los hechos que en ellas se omiten, ocultan y/o exaltan. 
Además, las autoras subrayan la importancia de tener una mirada crítica sobre los múltiples contextos que atraviesan las fuentes, incluyendo las condiciones sociales e históricas de producción -tales como las situaciones de enunciación y las tensiones entre lo oficial y lo particular- y los supuestos de quien investiga.

En nuestro continente, esta discusión se vincula con el uso de los documentos elaborados por viajeros, misioneros y funcionarios en base a objetivos propios relacionados con el conocimiento y posible dominio de grupos nativos (Nacuzzi y Lucaioli, 2011). Por ello, el reconocer en estos materiales datos relativos a las poblaciones que ya habitaban América requiere un ejercicio de desandar los múltiples discursos que los atraviesan, con una mirada atenta no sólo a lo que las fuentes informan sino también a aquello que omiten.

En cuanto a la fuente que resulta de los testimonios orales, esta refiere a la presencia de la memoria y de la subjetividad por lo que conlleva procesos de resignificación y selección de acontecimientos en función del presente (Bertaux, 1993). Es importante recordar que todo relato es elaborado de acuerdo con marcos sociales, constituyendo un producto imbricado en convenciones históricas específicas (Halbwachs, 1950). Por estos motivos, el criterio de verdad que se construye mediante las fuentes orales refiere a la interpretación de los hechos y no a su reconstrucción (Guadarrama Olivera, 1990). Existe consenso en que incluir esta línea de indagación en la investigación histórica permite recuperar la experiencia de los actores sociales y el modo en que éstos construyen sus visiones -sobre el paisaje y el pasado- en relación con el contexto socio-histórico del que forman parte (Whiteley, 2002). En arqueología, las fuentes orales permiten generar una perspectiva integral de las prácticas sociales abordadas y reconocer procesos de memoria en torno a los materiales arqueológicos (Van Dyke y Alcock, 2003; entre otros). Como se verá más adelante, en el caso que aquí se presenta, las fuentes orales remiten a la experiencia de los entrevistados con los materiales arqueológicos y no al pasado prehispánico en que estos últimos fueron elaborados.

Sin ánimos de reducir las diferencias metodológicas que el análisis e interpretación de múltiples fuentes conlleva, subrayamos que gran parte de las mismas son construidas en relación con las preguntas de investigación (Bourdieu et al., 1973). En este caso, organizamos y analizamos las fuentes según su contexto de producción: arqueológico, geográfico, documentos de los siglos XVIII y XIX y testimonios orales. Entre las fuentes escritas analizamos: documentación -publicada e inédita- que refiere al estudio de materiales arqueológicos hallados en la región; documentos oficiales sobre la frontera; memorias institucionales y cartografía de distintos momentos históricos. Debido a su amplio rango temporal, consideramos estos materiales teniendo en cuenta las diferentes escalas temporales involucradas -de procesos poblacionales a trayectorias personales.

Por su parte, los testimonios orales fueron constituidos mediante entrevistas abiertas realizadas a pobladores locales adultos -residentes de áreas urbanas y rurales, docentes rurales, artesanos, aficionados de la historia local, coleccionistas, gestores de la cultura y la historia local-. En total realizamos 39 entrevistas y se trabajó con 26 personas. Estas tuvieron lugar entre los años 2012 y 2018, en el marco de un proyecto que aborda la apropiación de lo arqueológico en la microrregión del río Salado (Salerno, 2016). Un primer análisis fue presentado en Escosteguy et al. (2015). En este trabajo profundizamos esas interpretaciones. 
En todos los casos, el material relevado remite indirectamente a los momentos prehispánicos que investigamos. Con el estudio de estas múltiples fuentes generamos una perspectiva amplia del problema de investigación que nos ocupa. Principalmente, porque ofreció información tanto en términos de descripción y transformación del mundo del pasado, como en lo referido a los sentidos en torno a dicho pasado que forman parte del contexto contemporáneo. A continuación, presentamos las fuentes relevadas y la información generada en cada caso.

\section{Estudios arqueológicos en arroyo El Siasgo}

La información arqueológica generada para la microrregión del río Salado hasta la década de 1980 es escasa. Los primeros estudios giraron en torno a las propuestas de Florentino Ameghino respecto de la antigüedad de las ocupaciones humanas en América del Sur (Ameghino, 1884; [1880] 1918). En 1910, en el marco de una expedición impulsada por el Museo Nacional de Buenos Aires, Carlos Ameghino halló en El Siasgo un esqueleto humano. Sobre la base de la morfología craneana y de algunos huesos de las extremidades inferiores de este individuo, Florentino Ameghino (1910) propuso que se trataba de una nueva especie homínida que denominó Homo caputinclinatus. Sus interpretaciones fueron presentadas en el Congreso Científico Internacional Americano de 1910 y discutidas por varios académicos de la época que accedieron a los restos u otros con quienes Ameghino mantenía intercambios epistolares muy fluidos. Esta correspondencia se encuentra publicada en la colección dirigida por Alfredo Torcelli (Ameghino, 1936) y pudimos consultar los originales en el Archivo documental del MACN-CONICET. Allí se evidencia que a pocos días de realizar el hallazgo, Ameghino le escribía al Dr. Hermann von Iering del Museo Paulista de San Pablo describiendo el esqueleto, destacando su tamaño pequeño y adscribiéndolo a una especie intermedia entre $H$. pampaeus y $H$. sapiens (Ameghino, 1936). En el copiador del archivo accedimos a la memoria de 1910, donde se mencionan los trabajos de campo en el Siasgo como una de las "principales excursiones" del año.

Otros colegas que analizaron el hallazgo de Ameghino fueron Aldobrandino Mochi y Aleš Hrdlička. Estos investigadores señalaron que era un esqueleto de humano moderno cuyo cráneo había sido deformado culturalmente (Ameghino, 1934). Willis (1912), geólogo que acompañó a Hrdlička en su investigación, recorrió el sitio del hallazgo acompañado de pobladores locales que le brindaron información adicional sobre las condiciones contextuales del mismo. El registro fotográfico de esa visita nos permitió considerar cuestiones bioantropológicas del entierro (Escosteguy et al., 2017). A pesar de que las interpretaciones de Ameghino se refutaron, sus debates impulsaron los estudios arqueológicos y bioantropológicos en la región (Politis y Bonomo, 2011). No obstante, los trabajos que continuaron su camino fueron desplazando el interrogante sobre la antigüedad de los restos al problema de su distribución espacial (Podgorny, 2001).

Este es el caso de Félix Outes $(1898,1936)$, quien estudió materiales arqueológicos de la cuenca del río Salado provistos por coleccionistas locales. Sus interpretaciones articularon el estudio de los materiales con información de fuentes escritas, entre las cuales otorgó mayor importancia a los relatos de Ulrico Schmidl ([1534-1554] 1903), ${ }^{2}$ por tratarse de un cronista que relató hechos a partir de sus propias vivencias. Describió la procedencia de estos materiales en términos de "paraderos Querandíes". Además, en función de la densidad 
3. Puede consultarse Escosteguy et al. (2015 y 2017).

4. Para mayor discusión véase Frère (2015). de los hallazgos, Outes distinguió entre "paraderos momentáneos" y "verdaderos núcleos de ocupación". Entre estos últimos se encuentran lugares en los bordes de las lagunas de Chascomús, Lobos y Monte -y otras zonas cercanas a Buenos Aires.

Cuatro décadas más tarde, Mario López Osornio (1942) presentó un mapa con la posible ubicación de los "paraderos Querandíes". Al igual que Outes, este autor trabajó con materiales de colecciones privadas de la zona y para sus interpretaciones se basó en fuentes escritas. En 1969, Marta Pastore (1974) comienza a recorrer la cuenca inferior del río Salado, realizóando prospecciones y excavaciones en las lagunas Esquivel, Las Barrancas, El Cacique y Cerrito de La Reducción. En estos trabajos de campo, recuperó tiestos, materiales en piedra y escasos restos óseos. En su interpretación, la autora hace referencia a un "proceso de paraneolitización" protagonizado por indígenas que tenían una economía cazadora-recolectora y que eran nómades o seminómades -en momentos más recientes. En ambos casos, estos estudiosos estuvieron centrados en documentar secuencias histórico-culturales y zonas de influencias. Por estos motivos, tuvo mayor centralidad la pregunta por la distribución geográfica de los hallazgos.

En la década de 1980, nuestro equipo comenzó a desarrollar investigaciones sistemáticas en el área desde un marco procesual. Se abandonó la discusión respecto de la adscripción étnica y se focalizó en los procesos poblacionales prehispánicos a partir de las teorías de cazadores-recolectores (González, 2005; entre otros). Concretamente, en El Siasgo comenzamos a trabajar en 2013. ${ }^{3}$ De forma resumida, se puede mencionar que se recuperaron numerosas evidencias arqueológicas en ambas márgenes del arroyo y en las lomadas loessicas asociadas. Los materiales incluyen tiestos cerámicos de distintas dimensiones, conjuntos líticos de lascas, artefactos confeccionados en rocas provenientes de las sierras bonaerenses y escasos materiales arqueofaunísticos. A partir de ellos se interpretó que el sector estuvo habitado durante el Holoceno tardío por grupos cazadores-recolectores y posiblemente pescadores (Escosteguy et al., 2015). Los fechados radiocarbónicos ubican los hallazgos en ca. 2100 años antes del presente, dando cuenta de una de las fechas más tempranas para la microrregión del río Salado. ${ }^{4}$ En complementación con este abordaje de los materiales, realizamos un análisis bioarqueológico del esqueleto hallado en 1910: se trata de un individuo juvenil con deformación craneana de tipo circular cuya dieta fue mixta, con consumo de recursos marinos en pequeñas cantidades. El fechado obtenido para este esqueleto es de ca. 3500 años AP (Escosteguy et al., 2017).

\section{Relaciones interétnicas en la frontera}

En uno de los documentos correspondientes a la Comandancia de Frontera de la Guardia del Zanjón de 1768, se relata la partida de un grupo de hombres "con el destino de reconocer el Parque que llaman las Islas y dan vuelta por el Ciajo, de cuyo Paraje acaban de llegar once hombres, los que descubrieron en una emboscada mucha yndiada". ${ }^{5}$ En principio, este constituye el primer registro que tenemos con la mención del paraje, tanto en documentos como en cartografía. En este se presenta una descripción de El Siasgo, en tanto territorio a explorar y de disputa. A su vez, se destaca el uso del término "islas" que también se registró en testimonios orales para describir las lomadas que no se anegan durante los períodos de crecidas. Por otro lado, el eje central de este informe nos remite a un territorio en el que tuvieron lugar contactos violentos 
entre indios e hispano-criollos. En el parte mencionado, el Comandante Juan de Mier señala la posibilidad de represalias y pide a las autoridades pólvora y balas para la defensa del Fuerte.

El segundo documento en el que registramos la mención de El Siasgo, corresponde a un expediente judicial de 1819. Se describe un conflicto que tuvo lugar en el paraje entre una familia de indígenas -"Indios Medinas"- y pobladores criollos de la zona. ${ }^{6}$ Este expediente da cuenta de la incorporación del territorio de El Siasgo en la administración colonial, lo que marca una diferencia fundamental respecto del parte de 1768, en el que El Siasgo aparece como un territorio a explorar. Los "Indios Medinas", llevaban alrededor de diez años asentados allí cuando son acusados por Don Ignacio Arista de perpetrar el robo de ganado. En los argumentos vertidos en este expediente se hace referencia a la economía de estos pobladores, dando cuenta de las regulaciones coloniales que limitaron ciertas actividades, marcando un giro en el modo de habitar y construir sentido sobre el paraje. Así, se describe negativamente el hecho de que los "Indios Medinas" excepcionalmente participaran del trabajo agrícola y, por estos motivos, los recursos vegetales y animales que poseían son señalados como evidencias de vandalismo. Estas argumentaciones invisibilizan otras posibles formas de obtención de dichos recursos, como por ejemplo la caza, recolección y pesca en un entorno de humedal caracterizado por su abundancia. Además, se ha documentado el intercambio de productos obtenidos mediante actividades cinegéticas -por ejemplo, cueros de coipos-, tanto en las pulperías instaladas en la línea de frontera o en las pulperías volantes que recorrían en carretas el extenso territorio de frontera (Mayo y Latrubesse, 1998). Diversas fuentes judiciales de espacios fronterizos fueron analizadas por Ratto (2005), quien destacó su valor para indagar aspectos relativos con las esferas laborales, las redes comerciales y las relaciones de parentesco y compadrazgo entre indios y criollos. Asimismo, esta autora observó que en el caso de los "Indios Medinas", los testimonios dejan entrever la presencia de relaciones consolidadas entre criollos e indígenas que involucraron matrimonios interétnicos y múltiples redes de reciprocidad.

Ambos documentos relevados remiten a distintos momentos de un particular proceso ocurrido en El Siasgo y que tiene que ver con su construcción como zona de frontera, ámbito de complejos intercambios culturales entre criollos e indígenas (Frère, 2000). Desde una perspectiva etnohistórica, se destaca la importancia geopolítica de este punto de la campaña bonaerense ya que, como menciona Vidal (1937), la desembocadura del arroyo en el río Salado era un lugar donde éste último podía vadearse fácilmente. Los documentos analizados dan cuenta de que en este paraje, al convertirse en frontera, se generaron nuevos términos para negociar actividades, formas de estar y construir sentido en torno al territorio, su pasado y su proyección. En el marco de relaciones asimétricas entre indígenas e hispano-criollos las estrategias extractivas, que por largo tiempo prevalecieron en este territorio, perdieron centralidad. Además, su descalificación nutrió representaciones estigmatizantes respecto de las poblaciones indígenas que en la actualidad continúan vigentes.

\section{Relevamiento cartográfico}

Debido a que el paraje El Siasgo ha sufrido modificaciones a lo largo del tiempo, otra línea de indagación que seguimos fue la revisión de cartografía elaborada en distintos momentos de los siglos XVIII, XIX y XX. Estos mapas revelan cómo se fue conociendo y administrando el territorio y nos permiten
6. AGN. Sala X, 11-3-3. 
identificar ciertos cambios y continuidades respecto de su ordenamiento y de los elementos que se consideraron significativos en diferentes momentos (Garavaglia y Gautreau, 2011). Las cartas relevadas se sintetizan en la Tabla 1.

\begin{tabular}{|l|l|l|}
\hline \multicolumn{1}{|c|}{ AÑO } & \multicolumn{1}{|c|}{ DENOMINACIÓN } & \multicolumn{1}{|c|}{ AUTOR } \\
\hline 1786 & Mapa geográfico & Custodio de Saá y Faria, en Torre Revello (1930). \\
\hline 1796 & Carta esférica de la Frontera del Sur de Buenos Ayres & $\begin{array}{l}\text { Pedro Antonio Cerviño y Juan Incyarte en Outes } \\
\text { (1930) }\end{array}$ \\
\hline 1821 & Plano geográfico que comprende la provincia de Buenos Ayres & J. M. Cabrer en Pesoa (2016: 279) \\
\hline 1864 & $\begin{array}{l}\text { Registro Gráfico de las propiedades rurales de la Provincia de Buenos } \\
\text { Aires construido por el Departamento Topográfico y publicado con } \\
\text { autorización del Superior Gobierno de la Provincia }\end{array}$ & $\begin{array}{l}\text { Saturnino Salas, Mariano Moreno, Germán } \\
\text { Kurn, Pedro Benoit, Ygnacio Casagemas y } \\
\text { Antonio Malaver }\end{array}$ \\
\hline 1870 & $\begin{array}{l}\text { Carta de una parte de la Provincia de Buenos Aires y las Pampas, con } \\
\text { demostración de la actual Línea de Fronteras, las proyectadas por el } \\
\text { Gobierno Provincial y el Congreso Nacional }\end{array}$ & Departamento Topográfico en Pesoa (2016: 269) \\
\hline $1901-1905$ & Atlas del plano catastral de la República Argentina & Carlos Chapeaurouge \\
\hline ca.1920 & $\begin{array}{l}\text { Colección de Planos Catastrales de los Partidos de la Provincia de } \\
\text { Buenos Aires. Construido con datos particulares, y confrontados con } \\
\text { la Estadística Oficial }\end{array}$ & Gregorio Edelberg \\
\hline
\end{tabular}

Tabla 1. Cartografía consultada en las que se representa el paraje El Siasgo.

En el registro más antiguo que analizamos -el mapa geográfico de 1786 (en Torre Revello, 1930)- aunque el sector de El Siasgo no se encuentra representado, aparece graficada la Guardia de Ranchos. Esto se puede contrastar con las referencias de la carta que confeccionan Cerviño e Incyarte en función de los datos relevados en 1796 por Félix de Azara, plasmados en su diario ([17421821] 1837). Este mapa presenta grandes diferencias de orientación y ubicación de la guardia de Ranchos respecto al confeccionado en 1786. En el diario y en el mapa del viaje de Azara, El Siasgo aparece mencionado como Ceajo. En su descripción, relata que saliendo de la Guardia de Ranchos atraviesan esta cañada y su laguna, además destaca que esta zona era más horizontal que lo observado en su camino desde la Guardia de Chascomús.

En la carta correspondiente a 1821 (en Pesoa, 2016: 279), que incluye una propuesta de división del área en cinco partidos, se observan grandes similitudes con el mapa descripto anteriormente. Asimismo, están señalados el arroyo y la laguna "de Ciajo". Al igual que en el mapa de 1796, se repite el error de la ubicación del arroyo Taqueño que corre muy próximo y paralelamente al arroyo El Siasgo. En ninguna de estas tres cartas aparecen nombrados pasos específicos.

En el mapa elaborado por Saturnino Salas y colaboradores en 1864 -véase detalle del sector estudiado en la Figura 1- ya se puede apreciar, entre otras cuestiones, el camino denominado "Camino al Monte" que cruza el arroyo El Siasgo. Este pasaje continuó siendo a lo largo de las décadas una vía muy efectiva para comunicar ambos sectores del paisaje, incluso hay referencias a accidentes que ocurrieron allí con carretas. Además, nos ofrece información referida a puntos en el espacio, tales como la Loma de Espinosa, lomada loessica así denominada porque se ubica en el campo cuyos propietarios eran los herederos de Tiburcio Espinosa. En este mapa también se ven los Pasos de Ponce y de Ramos del río Salado, muy próximos al arroyo El Siasgo. 


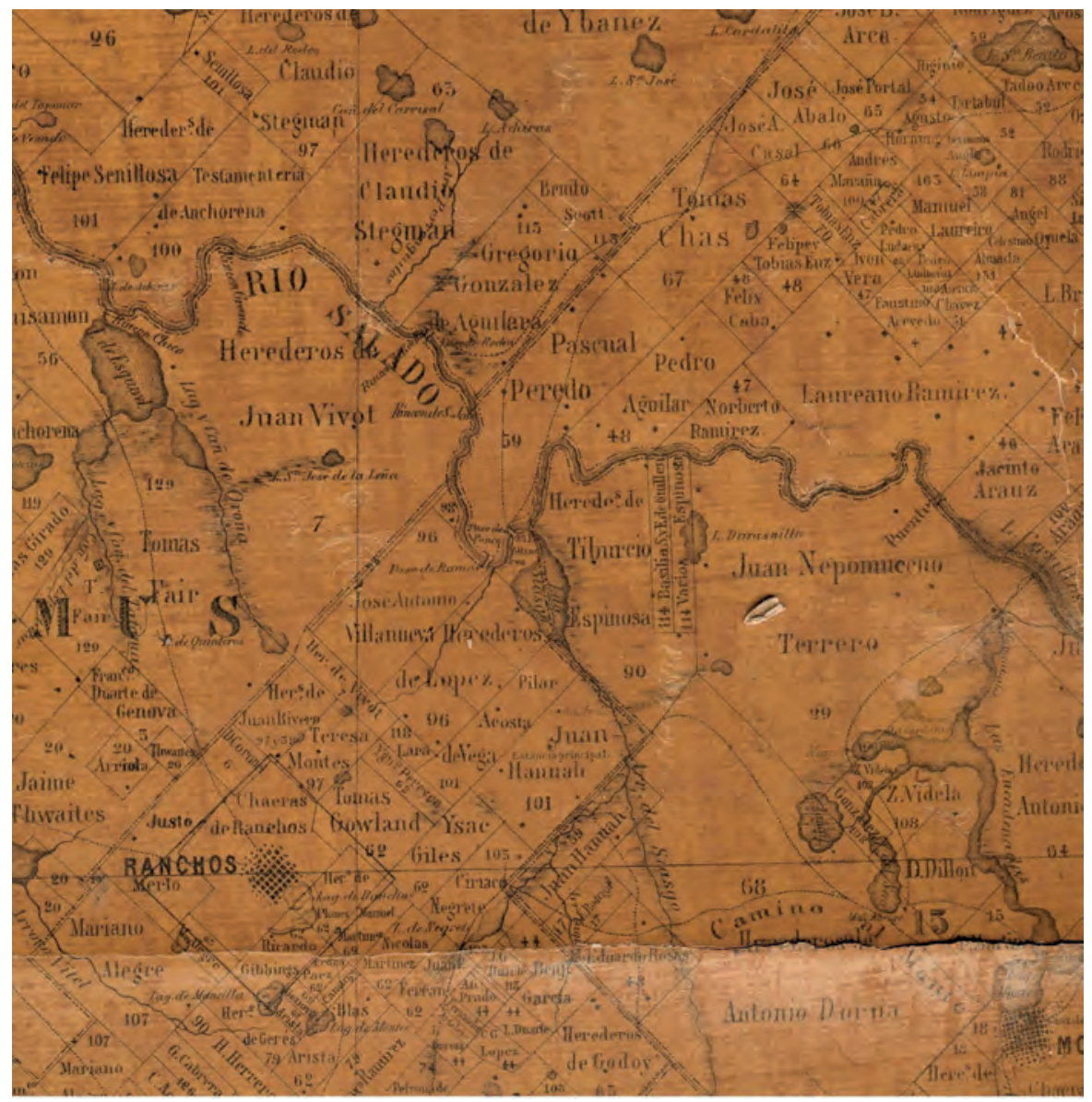

Figura 1. Detalle del sector de estudio en el mapa de 1864 elaborado por Saturnino Salas y colaboradores.

Este registro gráfico se publicó impreso en un grabado en seis hojas realizado por la Litografía del Departamento Topográfico. Se trata de un catastro de tierras de tipo jurídico, orientado a poner de manifiesto y garantizar la propiedad, su forma general, su ubicación y sus linderos (Favelukes, 2013). Por estos motivos, en estos mapas figuran los dueños de las parcelas, información que está vinculada con el interés de los propietarios e inversores nacionales y extranjeros de visualizar el estado de la tierra -incluida la situación de aquella en mano de indios- para plantear sus peticiones de posesión o de compra.

En el mapa de 1870 (en Pesoa, 2016: 269) no figuran los Pasos antes mencionados pero aparece el puente de Terreros como cruce más próximo río arriba. Por otro lado, ya se representan las vías del ferrocarril con las cabeceras en Chivilcoy, Lobos y Chascomús, sin llegar a Ranchos ni Villanueva -estaciones más próximas al sector de estudio.

A comienzos del siglo XX, Carlos Chapeaurouge (1901), sintetiza sus trabajos previos de agrimensura y topografía en el Atlas del plano catastral de la República Argentina. En él señala los distintos distritos, ciudades, pueblos, vías de circulación, cursos y cuerpos de agua, datos topográficos de relieve, entre otros detalles. En nuestra zona de estudio, registra "Arroyo del Siergo" (en lugar de Siasgo) y "Arroyo Pequeño" -en vez de Taqueño. En este mapa ya está documentada la ubicación de la estación de Villanueva del entonces Ferrocarril del Sud. 
Para la década de 1920, Gregorio Edelberg (ca. 1920) registra planos catastrales de los partidos de la Provincia de Buenos Aires. Aquí, está representado el Arroyo Siasgo y al igual que el mapa de Chapeaurouge (1901), están delineadas las estaciones del ferrocarril. Figuran también los propietarios de las distintas parcelas con algunas continuidades y diferencias en el registro de propiedad.

En suma, podemos destacar que la información obtenida del estudio de los mapas nos permitió, entre otros temas, indagar sobre las transformaciones del paisaje en diferentes temporalidades que incluyen cambios de la toponimia y la apropiación privada de la tierra. Tanto el arroyo El Siasgo como los pasos vinculados a su desembocadura han sido elementos que fueron reiteradamente mapeados dando cuenta de su importancia a lo largo del tiempo.

\section{Narrativas orales sobre el pasado prehispánico del arroyo El Siasgo}

Las narrativas orales relacionadas con el pasado indígena y con los materiales arqueológicos hallados en El Siasgo circulan principalmente entre personas que viven o han vivido en el paraje rural y/o en el pueblo de Villanueva. El análisis de las entrevistas se organizó a partir de ejes temáticos planteados inicialmente en esta investigación, relacionados con la presencia/ausencia de materiales arqueológicos y la temporalidad del poblamiento prehispánico de la zona, junto con otros tópicos que fueron incluidos por los entrevistados: como historias relativas a un mito sobre el poblamiento indígena y a la presencia/ ausencia de personas con ascendencia indígena en la actualidad.

En relación con los primeros ejes, la presencia de los materiales arqueológicos es mencionada mediante la referencia a materiales hallados en el campo y que en la actualidad se encuentran en contextos privados: principalmente bolas de boleadora y materiales de piedra pulida. Estos elementos son utilizados como objetos decorativos $\mathrm{y}$, en ocasiones, son regalados e intercambiados como "antigüedades". A pesar de que en esta zona rural también es común hallar fragmentos de alfarería y lascas, estos no suelen ser reconocidos como materiales arqueológicos y/o antiguos. Al punto que en una ocasión, al ver los fragmentos de lascas que habíamos recolectado durante el trabajo de campo, una pobladora rural nos dijo "¿esas piedritas de colores buscan? está lleno por acá, no sé decirte dónde porque están en todos lados". ${ }^{7}$ De igual forma, el puestero de un campo arrendado que previamente nos había mostrado una bola de boleadora y una punta de flecha que mantenía en su poder, al ver las lascas expresó su sorpresa respecto de qué era lo que buscábamos en el campo. Este entrevistado, con quien nos encontramos en distintas ocasiones, se mostró incrédulo frente a la información de los fechados arqueológicos, al punto que lo discutió con sus conocidos: le resultaba muy extraño que esas pequeñas piedras pudieran ser antiguas y de manufactura humana.

Particularmente, los objetos seleccionados como arqueológicos por estas personas son asociados con la historia indígena, y en ocasiones interpretados a partir de fuentes historiográficas y/o las narrativas arqueológicas elaboradas en la primera parte del siglo XX. A modo ilustrativo, en la Figura 2 se observa la representación de los antiguos pobladores del río Salado que se distribuye en el libro de Antonio Moncaut (1966: 9). Este historiador, ampliamente conocido en la zona, difunde en su libro la teoría ameghiniana sobre la existencia del hombre terciario en la región. Su uso local focaliza más en 
la representación de lo indígena en clave natural y menos en la profundidad temporal del poblamiento.

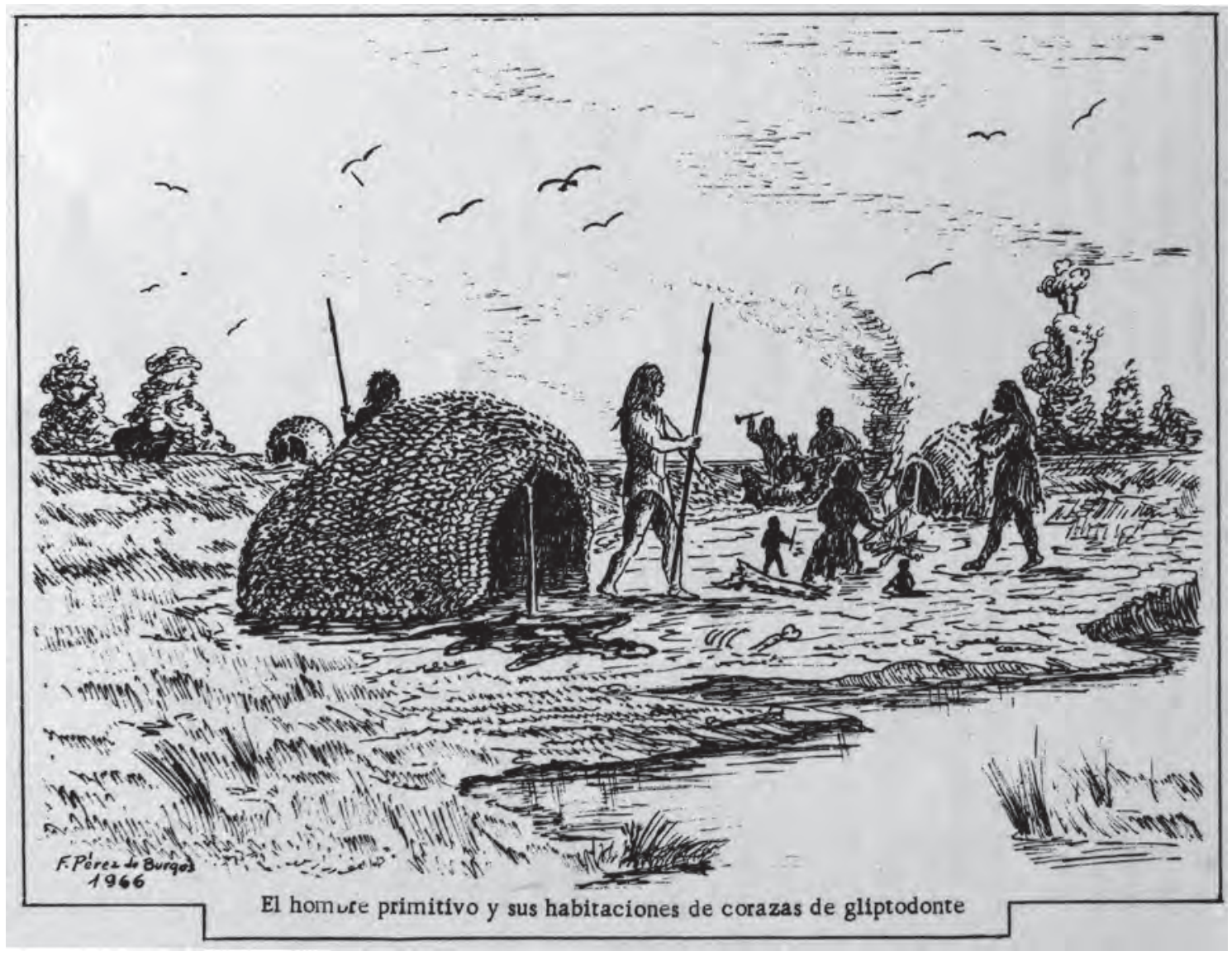

Figura 2. Representación de los antiguos habitantes presentada en Moncaut (1966: 9).

A su vez, la historia indígena es asociada con lugares específicos del paisaje: la desembocadura del arroyo El Siasgo en el río Salado; las lomadas y bosques de tala que caracterizaron esta zona de la campaña bonaerense-llamadas islas por los lugareños de antaño- y las lagunas temporarias que surcaban el paisaje: "dicen los antiguos pobladores que los pampas le llamaban las siete lagunas [...] el paisaje cambió mucho con las dragas [...] antes, cuando llovía se llenaba de lagunas, pescabas en todos lados". ${ }^{8}$

Respecto de la temporalidad en que se ubican las historias referidas al poblamiento indígena, puede decirse que remite tanto a un tiempo pasado impreciso, comprendido en las valoraciones de los objetos en términos de reliquia y antigüedad, como a la época de la frontera inferida a partir de las historias de indios que circulan -más adelante nos centraremos en la historia del cacique Siasgo. De este modo, se reproducen las narrativas de la historiografía tradicional que redujeron los procesos poblacionales prehispánicos del actual territorio bonaerense a las problemáticas de la "frontera interior" (Mandrini, 2007). En estas narrativas, la inclusión de los territorios indígenas al Estado nacional a fines del siglo XIX fue abordada como una gesta civilizadora. A pesar de los consensos elaborados desde la historia, la arqueología y la etnohistoria que documentan la complejidad y profundidad temporal de los procesos
8. Entrevista con docente y habitante rural, 27 de marzo de 2013 
9. Ver Nacuzzi (2005) y Mandrini (2007), entre otros.

10. Entrevista con poblador rural, 17 de mayo de 2015 .

11. Entrevista con docente rural, 29 noviembre de 2013.

12. ACCPS. Acta Fundacional del campo de Pato El Siasgo, 1954.

13. Entrevista con miembro del Club, 28 de octubre de 2014 poblacionales de esta región, ${ }^{9}$ la invisibilización y la estigmatización de las poblaciones indígenas continúan vigentes. En este caso de estudio, se manifiesta mediante la negación y/o desconocimiento de la profundidad temporal del poblamiento prehispánico. Lejos de tratarse de un caso aislado, este tipo de representaciones forman parte de los mitos fundadores en otras localidades de la microrregión (Salerno, 2014).

En contraparte, ciertos elementos que surgieron en las entrevistas nos llevan a considerar que en El Siasgo el pasado indígena se recupera como un elemento fundamental de la historia del lugar y de identificación territorial. En este camino interpretamos las historias sobre el cacique Siasgo, que más de un entrevistado puntualiza con validez relativa pues "no está bien claro por qué el Siasgo tiene un nombre Querandí". ${ }^{10}$ Esta explicación algo difusa refiere a la existencia de un cacique llamado Siasgo y su familia, quienes habían ocupado la zona en tiempos de "lucha con la civilización"11 y que a pesar de vivir pacíficamente a orillas del arroyo fueron asesinados por los pobladores hispanos. Dicha historia, que se transmite de forma oral e informal para fundamentar un origen indígena del topónimo Siasgo, forma parte del imaginario compartido respecto del territorio. Al punto que fue enseñada en la escuela del paraje -Escuela Rural № 8 El Siasgo-, como parte de la historia local e inclusive fue recreada en actos escolares. Aunque varios de los entrevistados señalan a la escuela como un lugar central en la trasmisión de esta historia, no hemos registrado que la misma forme parte de los contenidos que se trabajan en la actualidad. Además, el relato acerca de este cacique se reproduce como curiosidad turística del partido de San Miguel de Monte, desde donde se invita a los visitantes a conocer el "rancho El Siasgo" -ubicado sobre la ruta provincial $\mathrm{N}^{\circ} 41$ a la altura del paraje- en el que se ofrecen a la venta artículos regionales. Con referencia al lugar, los folletos turísticos indican "Siasgo era el apellido de un cacique que fue asesinado y su cuerpo fue arrojado a un arroyo, por esta razón este curso de agua recibió el nombre de este indio" (CITAB, 2003).

Otras voces ponen en duda el mito del cacique Siasgo a partir de la referencia a un posible origen español del vocablo. Al respecto, Felipe A. García (1985) realizó una exhaustiva búsqueda, en pos de identificar el origen y transformación del vocablo, que incluyó junto con el rastreo bibliográfico, la observación de mensuras y la consulta a historiadores locales. Este estudioso utilizó como primer registro del topónimo el diario de Félix de Azara ([1742-1821] 1837). Descartó un posible origen europeo o araucano del vocablo, así como su relación con fecha conmemorativa, leyenda o anécdota lugareña y hecho histórico particular. En contraparte, postuló que el topónimo puede vincularse con una voz tehuelche que perteneció a un personaje nacido en Tapalqué, a $120 \mathrm{~km}$ del paraje (García, 1985).

Con todo, llama la atención que a pesar de ser una historia cuya veracidad es cuestionada "porque no hay nada escrito", la misma continúa vigente trayendo a un primer plano el pasado indígena de la zona. En tal sentido, destacamos que el nombre del club de Pato El Siasgo de Villanueva fue seleccionado por "tratarse de un nombre regional"12 lo que contribuía a establecer una "identidad con el lugar". ${ }^{13}$ Por su parte, el destino trágico del cacique y su familia constituye una referencia fundamental para explicar el actual desconocimiento de poblaciones indígenas en la zona. Así, su ausencia se describe mediante el recuerdo de ciertos personajes que algunos entrevistados mencionan como indígenas -principalmente vinculados con la vida en el campo- y que vivieron dos o tres generaciones anteriores. Entre ellos, se reitera la historia de Eulogia 
"la baguala" Sierra, quien vivió en la zona siendo chasqui a principios del siglo XX.

\section{El Siasgo desde una perspectiva integradora}

A modo de recapitulación, señalamos algunos de los vínculos identificados entre los registros presentados en los apartados previos. Cabe destacar que éstos constituyen un conjunto variado de información que articula diferentes escalas temporales y espaciales: representaciones cartográficas relacionadas con la exploración y la apropiación privada del espacio; la articulación de saberes arqueológicos asociados a procesos poblacionales que tuvieron lugar en dicho territorio; historias de vida cuya memoria se asienta en la toponimia local y en historias orales. Desde un enfoque territorial, estos registros nos ofrecen huellas sobre las transformaciones de El Siasgo como un espacio construido política y estratégicamente a lo largo del tiempo (Lefebvre y Enders, 1976). Este proceso es el resultado de la confluencia de múltiples prácticas vinculadas con las formas de habitar y usar la tierra, el desarrollo de mensuras y catastros y la elaboración de representaciones (Soja, 1985). Entre ellas, se destaca el impacto de las políticas estatales que, en distintos momentos, articularon saberes prácticos, administrativos y profesionales (Garavaglia y Gautreau, 2011).

Respecto de las formas de la apropiación de este paisaje en el pasado, la integración de las fuentes presentadas nos permite identificar la relevancia de algunos lugares así como la vigencia de la toponimia empleada para el paraje. Por ejemplo, el camino que cruza el arroyo El Siasgo, identificado desde el mapa de 1864, es también señalado en las entrevistas como una vía importante que comunica distintos sectores del paisaje y en la que pervive la memoria de un accidente de carreta. De igual forma, en las entrevistas se referencia un punto del paisaje como lugar de posibles hallazgos arqueológicos: la "loma de Espinosa", lomada que se visibiliza durante momentos de inundación. A partir de los mapas, entendemos que este nombre haría referencia a antiguos propietarios cuya presencia en la zona se remonta por lo menos a 1864. Otro ejemplo, son los pasos del río que se ubican en la desembocadura del arroyo El Siasgo, cuya presencia temprana en la cartografía está en consonancia con las interpretaciones etnohistóricas que señalan su importancia geopolítica. Especialmente, estas últimas referencias nos llevan a reflexionar sobre cuál pudo ser el rol de los pasos del río y la "loma de Espinosa" en este territorio en tiempos prehispánicos.

Por su parte, los diferentes registros analizados también dan cuenta de cómo, a lo largo del tiempo, se construyeron y redefinieron ciertas representaciones de alteridad en las que se ubica al pasado indígena prehispánico. Por un lado, hasta la década de 1980, los materiales hallados en la zona se agruparon de acuerdo con similitudes y diferencias técnicas que, a su vez, fueron identificadas con distintos grupos étnicos. Estos primeros abordajes se caracterizaron por una perspectiva etnocéntrica que interpretó las características de los objetos tomando como referencia la información disponible en documentos elaborados por viajeros del siglo XVIII y XIX. Los principales interrogantes abordados en esta primera etapa de la arqueología refirieron a la adscripción temporal y étnica de las poblaciones prehispánicas. El debate giraba entre quienes, por un lado, defendían una gran antigüedad para el poblamiento en la región -como Ameghino (1918(año)-; quienes les adjudicaban una antigüedad relativa más reciente (neolítico) -como Outes (1936año) y López Osornio (1942año) - y 
quienes reducían el proceso poblacional a tiempos inmediatamente anteriores a la conquista -como Hrdlička (1912).

En todos los casos, se construyó el consenso de que la microrregión estuvo poblada por pueblos llamados querandí. La diferencia en esta adscripción étnica fue que, para las posturas que defendían la profundidad temporal de la ocupación, los querandí eran la corriente poblacional menos antigua, entonces no todos los vestigios materiales podrían vincularse con dicho pueblo (Outes, $1898 ; 1936)$. En contraste, para quienes sostenían un poblamiento tardío todos los materiales de la microrregión podrían adscribirse a los querandí y ser interpretados a la luz de las crónicas y relatos de viajeros. En estas discusiones, la diversidad cultural era entendida para los primeros como un indicador de la profundidad temporal mientras para los segundos se relacionaba con diferentes formas de vida (Podgorny, 2001).

Así, las categorías utilizadas contribuyeron a sostener representaciones de alteridad que se remontan a las disputas documentadas en los siglos XVIII y XIX. Las transformaciones propiciadas en los espacios fronterizos de esta región no sólo se relacionaron con el uso del espacio sino que tuvieron un fuerte impacto simbólico. En consecuencia la historia indígena se situó, en algunos casos, en un tiempo lejano y distante mientras que, en otros, se redujo el proceso poblacional al tiempo del contacto colonial. A su vez, estas formas de construir conocimiento sobre el pasado indígena se articularon con políticas estatales que promovieron procesos de patrimonialización de los referentes materiales y desligaron el pasado indígena de la narrativa histórica provincial (Mandrini, 2007). Tal como se desprende de los testimonios orales, estas narrativas son ampliamente conocidas a nivel local y constituyen importantes referentes que organizan las representaciones de los objetos arqueológicos con lo indígena y en asociación con lugares específicos de la campaña bonaerense -bordes de ríos y lagunas-, entre ellos el paraje El Siasgo.

Otro punto a observar es que las fuentes orales relevadas remiten a la experiencia de los entrevistados con los materiales arqueológicos y no al pasado prehispánico en que dichos materiales fueron elaborados. Así, Guadarrama Olivera (1990) destaca el potencial de abordar este tipo de fuentes en términos de "testimonio indirecto", que pone de relieve lo que se transmite como parte de la tradición oral sobre un pasado lejano. Desde la antropología, estos aspectos se han discutido especialmente en su relación con procesos de memorias en diferentes temporalidades (Van Dyke y Alcock, 2003; Ramos, 2011). En estos sentidos, la memoria es asumida como un proceso situado que involucra la valoración selectiva de eventos del pasado junto con la interpretación creativa desde el presente (Ramos, 2011: 134). Por estos motivos, estas interpretaciones nos permiten articular la relación pasado-presente de los procesos del pasado investigado, en tanto dan cuenta de los cambios en su forma de representaciones. En este caso, resultan llamativas las resignificaciones locales de las narrativas hegemónicas del pasado prehispánico de la zona, así como la reivindicación de algunos objetos arqueológicos con sentidos de pertenencia a lugares específicos del paisaje local. De este modo, El Siasgo se constituye como un lugar de anclaje que remite al pasado indígena regional. Así interpretamos la asociación entre los objetos arqueológicos y la historia del cacique Siasgo como un soporte para la memoria "subterránea" -en términos de Pollack (2006)- del pasado indígena. Estas prácticas, ligadas a la valoración de los objetos arqueológicos nos interpelan al momento de considerar las narrativas que construimos desde la arqueología. 
Finalmente, desde una perspectiva arqueológica, el análisis de la cartografía también nos permitió avanzar en la ubicación de posibles sitios. Este es el caso de la identificación del campo donde se encontró el esqueleto humano en 1910, y cuyos registros encontramos desde ca. 1920 bajo el nombre de Sara Delfino de Méndez (Edelberg, ca. 1920). Esta familia es mencionada en las publicaciones de Ameghino (1910), Hrdlička (1912) y Willis (1912). Otro ejemplo es el de la posible ubicación del asentamiento de los llamados "Indios Medina". En el mapa de 1864, el de Chapeaurouge (1901) y el de Edelberg (ca. 1920), figura el apellido Arista en algunos campos de las cercanías de Ranchos. Este apellido también se menciona en el expediente para referir a un hacendado colindante al asentamiento de los Medina. De ser descendientes de los vecinos originales, esto contribuiría a estimar la ubicación del asentamiento.

\section{Palabras finales}

Nuestro objetivo al relevar estas diferentes fuentes de conocimiento fue ponderar en qué medida su evaluación integrada enriquece nuestra perspectiva de los problemas arqueológicos que estamos abordando. De su análisis, generamos información sobre aspectos relacionados con las características de los materiales arqueológicos; especialmente su distribución y la potencial ubicación de sitios. Además, la información elaborada da cuenta de las diversas trayectorias poblacionales que se entrelazan en El Siasgo y que, en parte, son referidas en las representaciones que se construyen sobre los objetos arqueológicos. A su vez, estas fuentes nos llevaron a pensar en El Siasgo como un espacio en el que tuvieron lugar procesos históricos marcados por la transformación y permanencia de prácticas y narrativas. Entre ellas, subrayamos las formas de circulación en el paisaje así como ciertas continuidades respecto de los sentidos otorgados a algunos puntos del mismo.

Gran parte de las fuentes indagadas incluyen referencias indirectas al pasado prehispánico, lo que nos permite generar información sobre cómo dicho pasado forma parte del presente siendo objeto de apropiación y resignificación social. De este modo, buscamos dar centralidad a sujetos y agencias -incluyendo entre ellos a la arqueología- que desde el presente confluyen en la construcción de múltiples sentidos sobre el pasado investigado. Por estos motivos la integración de estos registros, teniendo en cuenta sus diferencias, nos ayuda a construir nuestro problema de investigación, aportándonos variadas perspectivas sobre un mundo que nos es ajeno. Miradas construidas desde lugares distantes a dicho mundo y en algunos casos -como el de las narrativas arqueológicas-, desde lugares próximos a nuestro contexto de investigación.

\section{Agradecimientos}

A aquellas personas que participaron de las entrevistas. Al personal del Museo de Ciencias Naturales Bernardino Rivadavia (MACN-CONICET); Ignacio Legari (Jefe División Colección Museológica, Archivos y Conservación de Exhibiciones). Al Prof. Alejandro Fernández, por la revisión del abstract; a las Dras. Romina Frontini y Laura Quiroga y los evaluadores por sus comentarios críticos. Las investigaciones fueron financiadas por UBACyT $20020170100525 B A$, PICT 2015-0272 y PICT 2016-0368. 


\section{Fuentes documentales citadas}

" Archivo General de la Nación, Argentina (AGN), Sala IX. 1-5-3 Comandancia de Frontera. Parte de la Guardia del Zanjón de 1768.

» Archivo General de la Nación, Argentina (AGN), Sala X. 11-3-3 Guerra. Comandantes de Campaña y Frontera. Expediente judicial de 1819 sobre los indios Medinas.

»Archivo documental del Museo de Ciencias Naturales Bernardino Rivadavia (MACNCONICET): Correspondencia de Florentino Ameghino entre los años 1909 y 1911; Memoria institucional del año 1910.

" Archivo del campo y club de Pato Siasgo (ACCPS), Villanueva, General Paz, Buenos Aires, Acta Fundacional del campo de Pato El Siasgo. 1954.

» CITAB (2003). Centro de Investigaciones Territoriales y Ambientales Bonaerenses. "El Partido de Monte". Publicación en CD elaborada por el Centro de Investigaciones Territoriales y Ambientales Bonaerenses. Buenos Aires, Banco de la Provincia.

» INDEC (2012). Censo nacional de población, hogares y viviendas 2010: censo del Bicentenario. Resultados definitivos. Buenos Aires, Instituto Nacional de Estadística y Censos. Disponible en Internet: https://www.indec.gob.ar/ftp/cuadros/poblacion/ cens02010_tomo1.pdf. Consultado el : 6 de diciembre de 2017 


\section{Q Bibliografía citada}

"Ameghino F. (1884). Filogenia. Buenos Aires, La Cultura Argentina.

»Ameghino F. (1910). Descubrimiento de un esqueleto fósil en el Pampeano superior del arroyo Siasgo. Separata del Congreso Científico Internacional Americano: 1-16. Buenos Aires, Imprenta y Casa Editora “Juan A. Alsina”..

»Ameghino, F. ([1880] 1918). La antigüedad del hombre en el Plata. Buenos Aires, La Cultura Argentina (2 tomos).

»Ameghino, F. (1934). “Descubrimiento de un esqueleto fósil en el Pampeano superior del arroyo Siasgo" en Torcelli A. (comp), Paleoantropología argentina. Obras Completas y correspondencia científica de Florentino Ameghino, XVIII: 409-413. La Plata, Taller de Impresiones Oficiales.

» Ameghino F. (1936). “Correspondencia científica” en Torcelli A. (comp.), Paleoantropología argentina. Obras Completas y correspondencia científica de Florentino Ameghino, XXII. La Plata, Taller de Impresiones Oficiales.

»Azara, F. de ([1742-1821] 1837). "Diario de un reconocimiento de las guardias y fortines, que guarnecen la línea de frontera de Buenos Aires, para ensancharla” en de Angelis, P. (comp), Colección de obras y documentos relativos a la Historia Antigua y Moderna de las provincias del Río de La Plata, VI. Buenos Aires, Imprenta del Estado.

» Bertaux, D. (1993). “De la perspectiva de la historia de vida a la transformación de la práctica sociológica” en Marinas, J. M. y C. Santamarina (eds.), La historia oral: métodos y experiencias: 19-34. Madrid, Debate.

» Bourdieu, P.; Chamboredon, J. C. y J. C. Passeron (1973). El oficio del sociólogo. Paris, Ecole Practice de Hautes Etudes.

"Chapeaurouge, C. de (1901). Atlas del Plano Catastral de la República Argentina. Buenos Aires, Eigendorf y Lesser.

»Edelberg, G. (ca. 1920). Colección de Planos Catastrales de los Partidos de la Provincia de Buenos Aires. Construido con datos particulares, y confrontados con la Estadística Oficial. Buenos Aires, G. Edelberg.

»Escosteguy, P.; Scabuzzo, C. y M. I. González (2017). Análisis bioarqueológico de los restos de Arroyo Siasgo, (supuesto Homo caputinclinatus de Ameghino 1910). Revista Argentina de Antropología biológica 19 (2).

» Escosteguy, P.; Salerno, V.; Granda, P. y M. Vigna (2015). Primeros resultados de las investigaciones arqueológicas en arroyo el Siasgo (Depresión del río Salado, Buenos Aires). Relaciones de la Sociedad Argentina de Antropología XL (2): 645-653.

» Favelukes, G. (2013). Notas para una historia territorial. Los mapas catastrales de Carlos de Chapeaurouge. Seminario de crítica 187: 1-12. Instituto de Arte Americano e Investigaciones estéticas.

» Frère, M. M. (2000). La cuenca del Río Salado: un territorio de contacto. Arqueología 10: 177-191.

»Frère, M. M. (2015). Tecnología cerámica de los cazadores-recolectores-pescadores de la microrregión del río Salado, Provincia de Buenos Aires. Tesis Doctoral. Buenos Aires, Facultad de Filosofía y Letras, Universidad de Buenos Aires. Disponible en Internet: $\quad$ http://repositorio.filo.uba.ar/jspui/bitstream/filodigital/2966/1/uba_ ffyl_t_2015_902988.pdfhttp://repositorio.filo.uba.ar/jspui/bitstream/filodigital/2966/1/ 
uba_ffyl_t_2015_902988.pdf. Consultada el: 11 de julio de 2016.

"Garavaglia, J. C. y P. Gautreau (2011). Mensurar la tierra controlar el territorio. América Latina, siglos XVIII y XIX. Rosario, Prohistoria Ediciones.

"García, F. (1985). El topónimo "Siasgo" y su posible raíz tehuelche. Revista Sapiens 5: 38-56.

»González, M. I. (2005). Arqueología de alfareros, cazadores y pescadores pampeanos. Buenos Aires, Sociedad Argentina de Antropología. (Colección tesis doctorales).

" Guadarrama Olivera, H. (1990). Historia Oral: Usos y Abusos. Estudios sobre las Culturas Contemporáneas III (9): 69-76. México, Universidad de Colima.

» Halbwachs, M. (1950). La memoria colectiva. Zaragoza, Prensas Universitarias de Zaragoza.

" Hrdlička A. (1912). Early Man in South America. Bulletin 52: 269-272. Washington, Smithsonian Institution, Bureau of American Ethnology.

»Lefebvre, H. y M. J. Enders (1976). Reflections on the politics of space. Antipode A Radical Journal of Geography 8 (2): 30-37.

" López Osornio, M. A. (1942). Paraderos Querandíes. Contribución al estudio de la historia de los indígenas del país. Cuaderno Folklórico 5: 2-45.

" Mandrini R. (2007). La historiografía argentina, los pueblos originarios y la incomodidad de los historiadores. Quinto Sol 11: 19-38.

» Mayo, C. y A. Latrubesse (1998). Terratenientes, soldados y cautivos. La frontera, 1736-1815. Buenos Aires, Editorial Biblos.

»Moncaut, C. A. (1966). Biografía del río Salado de la provincia de Buenos Aires. La Plata, CEILP.

"Nacuzzi, L. (2005). Identidades impuestas. Buenos Aires, Sociedad Argentina de Antropología.

"Nacuzzi, L. y C. Lucaioli (2011). El trabajo de campo en el archivo: campo de reflexión para las Ciencias Sociales. Publicar 10: 47-62.

»Outes, F. (1898). Etnografía Argentina. Segunda contribución al estudio de los indios Querandíes. Buenos Aires, Imprenta de M. Biedma e Hijo.

»Outes, F. (1936). Una luz sobre los Querandi. Comunicación a la Sociedad Argentina de Antropología. Buenos Aires, Imprenta y casa editora CONI.

"Pastore, M. (1974). "Yacimientos arqueológicos de la cuenca del río Salado y del río Matanzas (provincia de Buenos Aires, República Argentina)". Actas del III Congreso Nacional de Arqueología y IV Encuentro de Arqueología del Litoral: 420-431. Montevideo, Centro de Estudios Arqueológicos.

»Pesoa, M. (2016). “Una ciudad para la Pampa. La construcción del territorio en la Provincia de Buenos Aires (1810-1916)". Barcelona, Tesis Doctoral, Departament d'Urbanisme i Ordenació del Territori, Universitat Politècnica de Catalunya.

" Podgorny, I. (2001). La clasificación de los restos arqueológicos en la Argentina, 18801940. Primera parte: La diversidad cultural y el problema de la antigüedad del hombre en el Plata. Saber y tiempo, Revista de historia de la ciencia 3 (12): 5-26.

"Politis, G. y M. Bonomo (2011). Nuevos datos sobre el "hombre fósil" de Ameghino. Publicación Especial de la Asociación Paleontológica Argentina 12: 101-119.

"Pollack, M. (2006). Memoria, olvido y silencio. La producción social de identidades frente a situaciones límite. La Plata, Ediciones Al Margen. 
»Quiroga, L. (2005). Disonancias en arqueología histórica: la experiencia del valle del Bolsón. Werken 7: 89-109.

»Ramos, A. (2011). Perspectivas antropológicas sobre la memoria en contextos de diversidad y desigualdad. Alteridad 21 (42): 131-148.

»Ratto, S. (2005). Rompecabezas para armar: el estudio de la vida cotidiana en un ámbito fronterizo. Memoria Americana. Cuadernos de Etnohistoria 13: 179-207.

»Salerno, V. M. (2014). Trabajo arqueológico y representaciones del pasado prehispánico en Chascomús. Buenos Aires, Editorial de la Facultad de Filosofía y Letras, Universidad de Buenos Aires.

»Salerno, V. (2016). Apropiación de objetos arqueológicos en la microrregión del río Salado bonaerense. Revista de Antropología del Museo de Entre Ríos 2 (2): 92-96.

»Salas, S.; Moreno, M.; Kurn, G.; Benoit, P.; Casagemas, Y. y A. Malaver (1864). Registro Gráfico de las propiedades rurales de la Provincia de Buenos Aires. Departamento Topográfico y Superior Gobierno de la Provincia. Lito. Rodolfo Kratzenstein, Buenos Aires.

»Schmidl, U. ([1534-1554] 1903). Viaje al Río de La Plata. Buenos Aires, Cabaut y Cía. Disponible en internet: http://www.cervantesvirtual.com. Consultado el: 1 de junio de 2008.

» Soja, E. (1985). "The spatiality of social life: towards a transformative theorisation" en Derek G. yJ. Urry (eds.), Social relations and spatial structures: 90-127. Londres, Macmillan.

» Torre Revello, J. (1930). La Fundación de Chascomús. Ensayo histórico. Buenos Aires, La Facultad.

»Van Dyke, R. y S. E. Alcock (2003). Archaeologies of Memory. Oxford, Blackwell.

»Vidal, A. (1937). Los orígenes de Ranchos, General Paz, 1771-1865. La Plata, Archivo Histórico de la Provincia de Buenos Aires.

»Whiteley, P. (2002). Archaeology and Oral Tradition: The Scientific Importance of Dialogue. American Antiquity 67 (3): 405-415.

»Wilkie, L. (2006). “Documentary Archaeology” en Hicks, D. y M. Beaudry (eds.), Cambridge companion to Historical Archaeology: 13-33. Cambridge, Cambridge University Press.

»Willis, B. (1912). "Observations on the Arroyo Siasgo find” en Hrdli ka, A., Early Man in South America. Bulletin 52: 269-272. Washington, Smithsonian Institution, Bureau of American Ethnology. 